\title{
Typical emotional expression in children's drawings of the human face
}

\author{
Eleonora Cannoni ${ }^{1}$ (D) Giuliana Pinto $^{2} \cdot$ Anna Silvia Bombi $^{1}$
}

Accepted: 26 February 2021 / Published online: 20 March 2021

(C) The Author(s) 2021

\begin{abstract}
This study was aimed at verifying if children introduce emotional expressions in their drawings of human faces, and if a preferential expression exists; we also wanted to verify if children's pictorial choices change with increasing age. To this end we examined the human figure drawings made by 160 boys and 160 girls, equally divided in 4 age groups: $6-7 ; 8-9 ; 10-11 ; 12-$ 13 years; mean ages (SD in parentheses) were: $83,30(6,54) ; 106,14(7,16) 130,49(8,26) ; 155,40(6,66)$. Drawings were collected with the Draw-a-Man test instructions, i.e. without mentioning an emotional characterization. In the light of data from previous studies of emotion drawing on request, and the literature about preferred emotional expressions, we expected that an emotion would be portrayed even by the younger participants, and that the preferred emotion would be happiness. We also expected that with the improving ability to keep into account both mouth and eyes appearance, other expressions would be found besides the smiling face. Data were submitted to non-parametric tests to compare the frequencies of expressions (absolute and by age) and the frequencies of visual cues (absolute and by age and expressions). The results confirmed that only a small number of faces were expressionless, and that the most frequent emotion was happiness. However, with increasing age this representation gave way to a variety of basic emotions (sadness, fear, anger, surprise), whose representation may depend from the ability to modify the shapes of both eyes and mouth and changing communicative aims of the child.
\end{abstract}

Keywords Emotional expression $\cdot$ Human face $\cdot$ Children's drawings $\cdot$ Development

\section{Introduction}

This study was aimed at verifying if children introduce emotional expressions in their drawings of human faces, even if not explicitly requested; if so, we wanted to verify if a preferential expression exists and if children's pictorial choices change with increasing age. Human beings are one of the earliest and most common subjects of children's drawings,

Eleonora Cannoni

eleonora.cannoni@uniroma1.it

Giuliana Pinto

giuliana.pinto@unifi.it

Anna Silvia Bombi

annasilvia.bombi@ fondazione.uniroma1.it

1 Department of Developmental and Social Psychology, Faculty of Medicine and Psychology, Sapienza University of Rome, Via dei Marsi, 78, 00185 Rome, Italy

2 Department of Education, Languages, Intercultures, Literatures and Psychology, Faculty of Psychology, University of Florence, Via Di San Salvi 12, 50135 Florence, Italy and the development of the human figure drawing has been extensively studied (Cox, 1993). Moreover, this kind of drawing has been the basis for a number of psychological instruments designed to assess children's intellectual maturity and emotional adaptation (see Matto, 2007 for a synthesis) as well as interpersonal relationships (e.g. Bombi et al., 2020; Rabaglietti et al., 2012). To these ends, various aspects of human figure drawing have been taken into account, such as visual realism and symbolic value of the shapes. However, children's depictions of facial expressions have not received the same attention that has been given to the completeness and accuracy of the entire figure, as well as other emotional cues.

In relatively recent years a number of studies have examined the development of children's ability to represent expressive faces, either explicitly requiring an emotional connotation or proposing an evocative theme (see Burkitt et al., 2019, Laghi et al., 2014; Pezzica et al., 2016; , as recent examples including references to other relevant papers). According to these studies some properties of drawings, such as figure size, shape, or color, do possess an emotional meaning that can be investigated systematically. 
A few studies, however, provided data about children's ability to represent facial expressions, and about the pictorial equivalents employed for this purpose. One of the first authors to deal empirically with these topics (about thirty years ago) was Golomb, who asked children from 1st to 6th grade of primary school to draw a happy, a sad, and an angry child (Golomb, 2004). She found that children modified the shape of the lips to differentiate the faces according to the required emotion. Simple lines (upward curved, downward curved or straight) were employed starting even in first graders to represent happiness, sadness and anger. Lines were sometimes substituted by surfaces in the drawings of older children: a moon slit (upward for joy and downward for sadness) and other forms for anger (such as undulating or zigzag shapes, or visible teeth). Children did not modify the region around the eyes as much as they did the mouth, and only from the third grade and beyond were diagonal eyebrows used to indicate anger.

The depiction of other basic emotions was also studied. Missaghi-Lakshman and Whissell (1991) asked children from 2nd, 4th and 7th grade to fill blank ovals in order to create (in random order) six faces: happy, sad, angry, afraid, surprised and disgusted. Subsequently, children themselves and adult judges were asked to assign the proper emotion to each face (again, randomly presented). Happiness and sadness were the more recognizable emotions, followed by anger and surprise, with an improved recognizability of three of them (happiness, anger and surprise) in the older group; fear and disgust were the less recognizable at all the ages being considered. The authors did not examine systematically the pictorial equivalents employed, but noted that children often depicted "widely accepted standard symbols" such as upturned mouth for happiness, tears for sadness, wrinkled brows, exposed teeth and slanted brows for anger, wide open eyes for fear or surprise, and protruding tongue for disgust.

Melike Sayil (2001), in a study with three groups of participants, respectively 4,8 and 10 years old, tried to explain why children rely on the mouth rather than on the eyebrows to represent emotions, as noted by Golomb (2004) and found by Sayil herself in previous studies summarized in the paper we are describing (Sayil, 2001). She found that children from 6 years on were able to copy oblique eyebrows in angry and sad faces, and from 8 years on they also selected oblique eyebrows in a construction task; however even the older children found it very difficult to draw convergent or divergent lines without a model. Moreover, for the surprised and the happy face children did not pay attention to eyebrow position, selecting only the correct conventional mouth (respectively, a circle and an upward pointing arc). Sayil (2001) concluded that a partial answer to her research question came from the difficulty of drawing convergent or divergent oblique lines (required to obtain, respectively, an angry or a sad expression of the eyes), or to notice the subtle difference in position between the relaxed eyebrows of a happy face and the raised eyebrows of a surprised face. Subsequent studies taking into account various pictorial cues that could be used to show a person's emotion (face expression, posture and context) demonstrate that happy and sad face expressions are the first to appear in preschoolers' drawings (see Brechet et al., 2007 as an example of this approach).

Overall, these studies showed that: 1) children can modify the shape of some facial areas in order to show basic emotional expressions; 2) in the case of happiness this is already true for the large majority of preschoolers, and the repertoire of depicted emotion widens during middle childhood, including all basic emotions; 3 ) children can rely on the mouth shape to depict some emotions even from an early age, while eyes and brows shapes are more difficult to reproduce.

These data broadly correspond to the children's emotional competence, and in particular to their increasing ability to perceive and recognize diverse facial expressions. Sensitivity to facial expression is present in infants, with a preference for smiling over neutral face through the first 7 months (Sugden \& Marquis, 2017) and a subsequent increased attention to fearful expressions which has been considered the beginning of the so called "negativity bias", i.e. the tendency to focus on negative social information and to behave accordingly (Vaish et al., 2008). Despite the obvious relevance of threatening stimuli such as unpleasant emotional expressions, and the number of studies demonstrating that a negativity bias does exist (especially in anxious adults), preferential attention has been found also for positive expressions (Becker et al., 2011). Life-span studies (Carstensen \& DeLiema, 2018). have also shown that a positivity bias also exists, and is related to motivations and goals more than to cognitive abilities. On the other hand, when children's ability to discriminate basic emotions increases (including the ability to distinguish real from pretend emotion, Sidera et al., 2013), the accuracy is greater for happiness while there is a general tendency to confuse negative emotions and misinterpret neutral expressions (Gross \& Ballif, 1991).The difficulty in discriminating between emotional expressions has to do with the components of the face that manifest them, since the facial area in which the most distinctive components are located varies with each emotion (Ekman \& Friesen, 2003). Experimental studies have confirmed that a happy expression can be easily identified even if one looks just at a smiling mouth, while other emotions require an inspection of (at least) the eyes' region, or of both eyes and mouth (Beaudry et al., 2014). Easy to identify, the smile carries many other advantages: when perceived in others it enhances their facial attractiveness (Golle et al., 2014) as well as recognition and memory of them (Becker \& Srinivasan, 2014); when effected on one's own face, it is considered an indication of attention breadth and flexibility (Johnson et al., 2010) especially when it appears in its Duchenne form, i.e. when it includes activation of the cheek 
raiser muscle besides that of the lips corner puller (Gunnery \& Ruben, 2016). For children, too, smiling faces are easier to remember (Meng et al., 2019), they promote social relationships (Song et al., 2016), and are read as indices of desirable qualities such as intelligence (Talamas et al., 2016).

The literature summarized above does not tell us (1) if children would introduce emotional expressions in their drawings of human faces when not explicitly requested to do so; (2) if there would be a preferred emotional expression, and what it would be; (3) if there would be any pattern of change associated with children's age.

Considering the importance of emotions in human life and the early ability to depict them on request, we expect that emotional indices would typically appear in a drawn face. Among all possible expressions, happiness is a great candidate, being vivid and socially significant, as well as easy to represent with the manipulation of a single shape, the mouth. However, we expect that by late childhood facial expressions would be more varied, thanks to children's larger pictorial resources (in particular their ability to work also on the eyes' shape (Golomb, 2004; Sayil, 2001), their increasing knowledge about emotions (Castro et al., 2016) and their desire to personalize their own drawings with a less usual look (Lau, 2020).

We are aware of a possible influence of gender, for two main reasons. First, gender differences have been found in emotion expressions for adults (LaFrance et al., 2003) and for children (Chaplin \& Aldao, 2013). Second, some scholars have begun to find gender differences in children's drawings of expressive human faces (e.g. Brechet et al., 2013; Tuman, 2015). However, as a first step in a new direction, we are not putting forward any hypothesis as regards gender, considering here a necessary preliminary step to verify the results in the entire group of participants and by age.

\section{Method}

\section{Participants and Procedure}

The examined drawings are part of a data-set of about 1000 cases, collected in four towns of central-southern Italy. In each town, one elementary and one junior high school serving families of middle and middle-low SES were selected. Children who were authorized by parents to participate came from Italian families, and can be considered representative of the provincial central-southern Italian culture. Children were contacted in their classrooms during the regular school hours; each child who agreed to participate was individually submitted to the Draw-A-Man test in a separate room provided by the school. The test was administered according to the instructions in the Italian adaptation (Polácek \& Carli, 1977), which requires drawing the entire male human figure as best as possible.
Given the exploratory nature of this study we reduced the drawings to be examined to a total of 320 , a reasonable number fort the non-parametric analyses we planned to perform. To this end we randomly selected the drawings of 10 boys and 10 girls per class from first to fifth grade of primary school (ages from 6 to 10 years) and from first to third grade of junior high school (ages from 11 to 13 years).

\section{Classification and Analysis of Data}

The drawn faces were examined by two judges with extensive expertise in the analysis of human figure drawings in order to verify the presence of an emotional expression or its lack. To prevent an interpretative attitude, the judges were given a list of possible shapes of mouth and eyes + eyebrows for six basic emotions (happiness, sadness, fear, anger, surprise, disgust), based on the shapes found by other scholars in children's drawings of emotions and those action units described in Ekman and Friesen (2003) that children could represent in simplified form (see online the ESM 1 and the ESM 2 where coding criteria are described and visualized).

The judges were asked to categorize the facial expression as one of the 6 emotions listed above or neutrality, and for each emotion to determine which were the relevant visual cues. For the latter the judges covered the eyes first and then the mouth (or vice-versa, to avoid a carry-over effect); if the emotion remained recognizable in one of these partial views they assigned the category "mouth" or "eyes", if not they assigned the category "mouth + eyes". The agreement between the judges about the depicted emotions was near to 97\%; the 10 cases of disagreement were solved by a third judge. Non parametric tests were used for the following comparisons: frequency of expressions (absolute and by age); frequency of visual cues (absolute and by age and expressions). To avoid small numbers per cell, the participants were collapsed in four age groups of 40 boys and 40 girls each $(6-7 ; 8$ $8 ; 10-11 ; 12-13$ years). The mean ages in months are as follows (standard deviation in parentheses): $83,30(6,54)$; $106,14(7,16) 130,49(8,26) ; 155,40(6,66)$.

\section{Results}

Table 1 presents an overview of the data distribution by expressions, visual cues and participants age.

As shown in the last section in Table 1 (bottom marginal totals), all the expressions except disgust were represented, albeit with very different frequencies $\left(\chi^{2}(5)=618,59\right.$; $p<.001)$. The categories of visual cues, also shown in the last section of Table 1 (side marginal totals), were also significantly different $\left(\chi^{2}(2)=107,12 ; \mathrm{p}<.001\right)$. 
Table 1 Absolute frequency of expressions by visual cues and participants age

\begin{tabular}{|c|c|c|c|c|c|c|c|c|}
\hline & & neutrality & happiness & sadness & fear & anger & surprise & Total \\
\hline \multirow[t]{5}{*}{ 6-7 years } & no cues & 1 & & & & & & 1 \\
\hline & eyes & & & & 3 & & & 3 \\
\hline & mouth & & 53 & 1 & & & 1 & 55 \\
\hline & $\begin{array}{l}\text { eyes+ } \\
\text { mouth }\end{array}$ & & 11 & 2 & 4 & 2 & 2 & 21 \\
\hline & Total & 1 & 64 & 3 & 7 & 2 & 3 & 80 \\
\hline \multirow[t]{5}{*}{$8-9$ years } & no cues & 2 & & & & & & 2 \\
\hline & eyes & & 1 & & 1 & 1 & 1 & 4 \\
\hline & mouth & & 41 & 1 & & & & 42 \\
\hline & $\begin{array}{l}\text { eyes+ } \\
\text { mouth }\end{array}$ & & 18 & 5 & 5 & 3 & 1 & 32 \\
\hline & Total & 2 & 60 & 6 & 6 & 4 & 2 & 80 \\
\hline \multirow[t]{5}{*}{$10-11$ years } & no cues & 6 & & & & & & 6 \\
\hline & eyes & & & 1 & 2 & & 1 & 4 \\
\hline & mouth & & 28 & 1 & 1 & 1 & & 31 \\
\hline & $\begin{array}{l}\text { eyes+ } \\
\text { mouth }\end{array}$ & & 27 & 2 & 2 & 3 & 5 & 39 \\
\hline & Total & 6 & 55 & 4 & 5 & 4 & 6 & 80 \\
\hline \multirow[t]{5}{*}{$12-13$ years } & no cues & 11 & & & & & & 11 \\
\hline & eyes & & & 1 & 1 & 1 & 2 & 5 \\
\hline & mouth & & 19 & 2 & & 1 & & 22 \\
\hline & $\begin{array}{l}\text { eyes+ } \\
\text { mouth }\end{array}$ & & 21 & 8 & 3 & 9 & 1 & 42 \\
\hline & Total & 11 & 40 & 11 & 4 & 11 & 3 & 80 \\
\hline \multirow[t]{5}{*}{ all ages } & no cues & 20 & & & & & & 20 \\
\hline & eyes & & 1 & 2 & 7 & 2 & 4 & 16 \\
\hline & mouth & & 141 & 5 & 1 & 2 & 1 & 150 \\
\hline & $\begin{array}{l}\text { eyes+ } \\
\text { mouth }\end{array}$ & & 77 & 17 & 14 & 17 & 9 & 134 \\
\hline & Total & 20 & 219 & 24 & 22 & 21 & 14 & 320 \\
\hline
\end{tabular}

The frequency of expressions by age (independently from visual cues) can be seen in the gray boxes at the bottom of each section

The frequency of visual cues by age (independently from emotions) can be seen in the gray boxes at the right side of each section
The gray boxes in Table 1 show the frequency of expression by age (bottom boxes of each section) and by visual cues (side boxes of each section). Comparing happiness vs. all the other expression (neutrality included) by age we see that happiness decreases significantly while the other expressions increase $\left(\chi^{2}(3)=19,14 ; p=.001\right)$. As per visual cues, we see that eyes only are rarely used at all ages, the use of mouth only decreases, with corresponding increases in the use of both visual cues (mouth + eyes). The $\chi^{2}$ calculated after collapsing the data for mouth only and eyes only shows that this increase is significant $\left(\chi^{2}(3)=20,13 ; p<.001\right)$.

The comparisons of visual cues by emotions were carried excluding the 20 cases of neutrality, and dichotomizing the data as follows: happiness vs. all other emotions and one region of the face (i.e.mouth only and eyes only) vs. two regions (i.e. mouth + eyes). The McNemar test for related samples yielded the following results: $6-7$ years: $p=.21$ n.s.; 8 9 years: $p=.004 ; 10-11$ years: $\mathrm{p}=.001 ; 12-13$ years: $p=.024$; all participants: $p<.001$. Data are shown in Fig. 1 .

Examples of Figures drawn by participants with each expression are visible online from ESM 2 to ESM 14.

\section{Discussion}

The aim of this study has been to verify whether children consider emotional expression an important feature of the human face, and possibly what such expression would be; we also wanted to verify whether the increasing ability to take into account more than a single visual cue affects children's pictorial choices. In the absence of any mention of expressions, nearly $94 \%$ of the children represented their drawn 


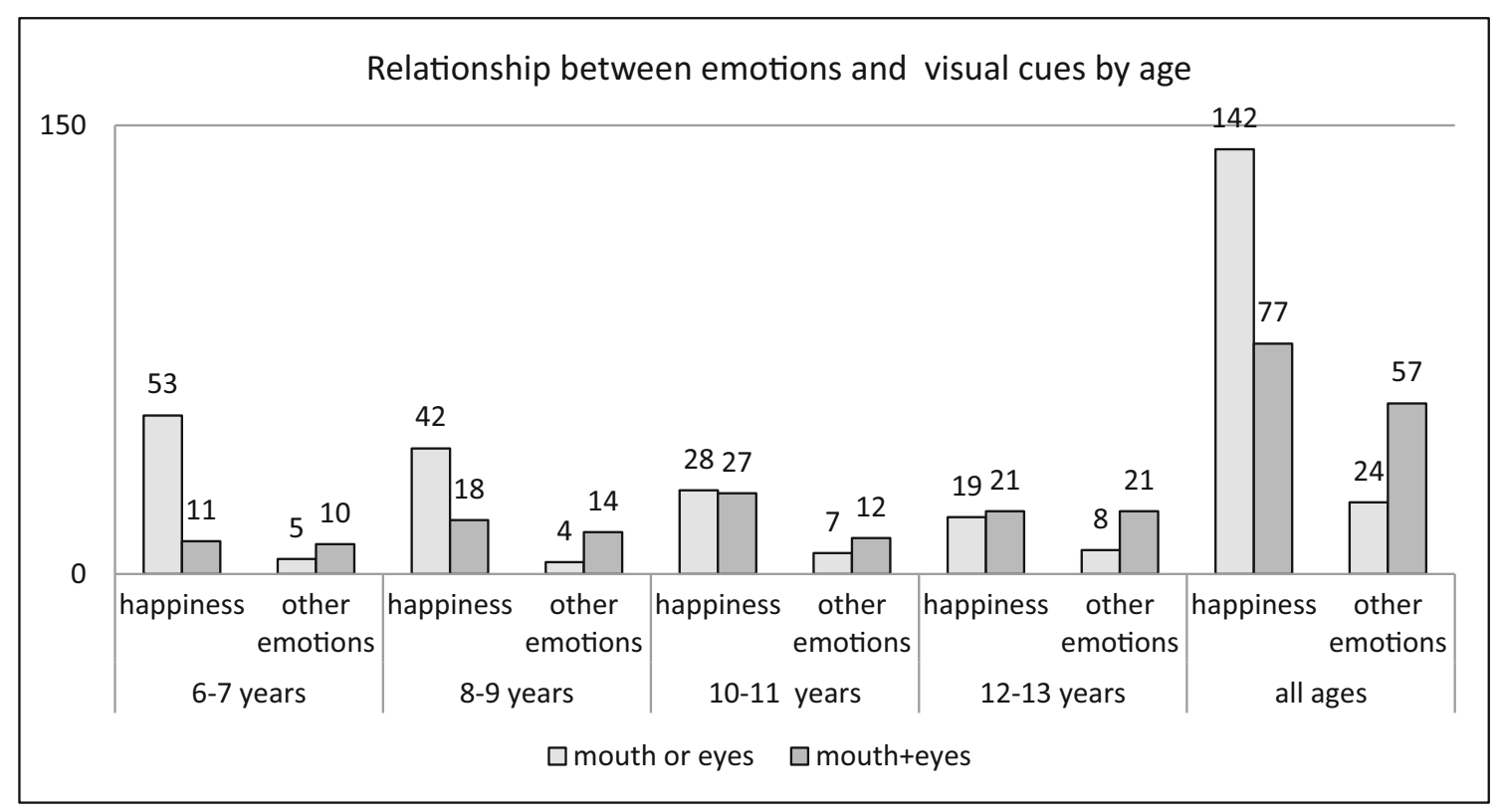

Fig. 1 Relationship between emotions and visual cues by age

faces with mouth and/or eyes shapes corresponding to that of a basic emotion. This is in line with our expectations and with the previous literature about children's ability to represent basic emotions on request (Brechet et al., 2007; Golomb, 2004; Missaghi-Lakshman \& Whissell, 1991).

From a methodological point of view, this result suggests the usefulness of complementing the study of knowledge and children's theories of mental life and emotional states with the use of productive drawing, alongside the methodology of visual recognition of represented emotions. The information we found could not be derived from the drawing tasks in which emotions are expressly required, nor from those in which emotions are evoked by an assigned theme, since both procedures are inevitably inductive to some extent.

It would also be appropriate to reconsider the weight of the facial details in the overall score of the Draw-a-Person test, which depends mainly on the correctness and number of anatomical parts, while it fails to acknowledge the ability to depict a human figure in what makes it such, that is, the emotional expressiveness. In other words, we suggest that the developmental tests based on the human figure drawing (Harris, 1963; Naglieri, 1988) have overestimated the role of conceptual thinking in drawing, and underestimated the role of intrinsic social-emotional organization, namely the early developmental need to make feelings visible in the drawings of human figures.

Since children's overwhelming preferences are for happiness, we can say that a smiling face is a substantial component of a canonical human figure, and might reveal how culturally appropriate appearances become part of children's representational goals. The preference for the happy expression has various reasons. First, as the data of Table 1 illustrate, the mouth alone is sufficient to show happiness (Beaudry et al., 2014) even if the addition of an appropriate shape of the eyes enhances the quality of smile (Gunnery \& Ruben, 2016; Song et al., 2016). Second, as shown in Fig. 1, a happy expression can be obtained modifying the shape of a single region of the face, which is not the case for the other emotions; we also know from the data in Table 1 that this single region is almost always the mouth. Last, but not least, the choice of a happy expression is highly conventional in western culture, since it corresponds to the numerous psychological advantages of smile, in terms of attractiveness (Golle et al., 2014), recognition and memory (Becker \& Srinivasan, 2014), and positive social and individual meaning (Talamas et al., 2016). In fact, sadness could be represented as easily as happiness, simply inverting the shape of the mouth curve, but a sad mouth appeared only 22 times (less than $7 \%$ of the drawings).

With age, however, the number of children who prefer other expressions increases (Table 1), including various basic emotions and neutral faces. Although drawing ability may contribute to the representation of more varied expressions in the face depiction, other emotion-related variables may intervene. The increase in less prototypical looks could be linked to a desire to make one's own drawing more personal and "unique" (Lau, 2020); to this end, less socially desirable appearances (e.g. angry, or fearful, or even emotionally detached) could be part of the need for greater expressive and emotional autonomy typical of preadolescents and adolescents.

Our data about the typicality of positive facial expression may be of interest from various perspectives. In clinical practice they suggest caution interpreting the emotions expressed 
by drawn figures. On the one hand, a smiling face should not be overestimated as a young child's intention to communicate positive moods, being the prevalent default at least until 89 years of age. On the other hand, a complementary caution is suggested by the presence (in a typical population) of a considerable number of negative emotions which almost double upon reaching adolescence. In a different vein, the preference for an expressive, smiling face could be useful in robotics, guiding the realization of simulacra as analogical as possible to the mental representations of the users and as such apt to promote their recognition.

Finally, we are aware of our study's limitations. First of all, a replication with a larger number of cases would be necessary to confirm, and eventually generalize the present results, as well as to verify if differences related to gender exist. The somehow unexpected proportion of preferences for a happy expression did not allow a fine statistical analysis of the other pictorial choices (other emotions or emotionless faces),. The lack of information about personal characteristics of the designers, such as creativity, visualmotor skills, but also temperament, did not permit an indepth interpretation of the individual pictorial choices of children who did not go for the usual happy expression. An examination of facial representations in the popular media (comic books, cartoons, and possibly street graffiti) could be of help in discussing the pictorial solutions adopted at various ages. It would also be interesting to extend the study to other cultures in which a smiling face has different meanings, as well as to atypical populations, and especially persons whose affective and communicative development is compromised, as in autism.

Supplementary Information The online version contains supplementary material available at https://doi.org/10.1007/s12144-021-01558-1.

Funding Open access funding provided by Università degli Studi di Roma La Sapienza within the CRUI-CARE Agreement.

Data Availability The data sets generated during and/or analysed during the current study are available from the corresponding author on reasonable request.

Code Availability Not applicable.

\section{Declarations}

Ethics Approval All procedures performed in this study were in accordance with the ethical standards of the institutional and national research committee and with the 1964 Helsinki Declaration and its later amendments or comparable ethical standards.

Consent to Participate Written informed consent was obtained from the parents and verbal consent from the children.

Consent for Publication Not applicable.
Conflicts of Interest/Competing Interests The authors have no conflicts of interest to declare that are relevant to the content of this article.

Open Access This article is licensed under a Creative Commons Attribution 4.0 International License, which permits use, sharing, adaptation, distribution and reproduction in any medium or format, as long as you give appropriate credit to the original author(s) and the source, provide a link to the Creative Commons licence, and indicate if changes were made. The images or other third party material in this article are included in the article's Creative Commons licence, unless indicated otherwise in a credit line to the material. If material is not included in the article's Creative Commons licence and your intended use is not permitted by statutory regulation or exceeds the permitted use, you will need to obtain permission directly from the copyright holder. To view a copy of this licence, visit http://creativecommons.org/licenses/by/4.0/.

\section{References}

Beaudry, O., Roy-Charland, A., Perron, M., Tapp, R., \& Cormier, I. (2014). Featural processing in recognition of emotional facial expressions. Cognition and Emotion, 28(3), 416-432. https://doi.org/ 10.1080/02699931.2013.833500.

Becker, D, V. \& Srinivasan, N. (2014). The vividness of the happy face. Current Directions in Psychological Science, 23(3), 189. https://doi. org/10.1177/0963721414533702, 194.

Becker, D, V., Anderson, U, S., Neufeld, S, L., Neel, R., \& Mortensen, C, R. (2011). The face in the crowd effect unconfounded: Happy faces, not angry faces, are more efficiently detected in single- and multipletarget visual search tasks. Journal of Experimental Psychology: General, 140(4), 637-659.https://doi.org/10.1037/a0024060.

Bombi, A, S., Cannoni, E., Gallì, F., \& Di Norcia, A. (2020). The relationship between teachers and students: Children's pictorial representation and teachers evaluation. Current Psychology. https://doi. org/10.1007/s12144-020-00702-7.

Brechet, C. (2013). Children's gender stereotypes through drawings of emotional faces: Do boys draw angrier faces than girls? Sex Roles, 68, 378-389. https://doi.org/10.1007/s11199-012-0242-3.

Brechet, C., Picard, D., \& Baldy, R. (2007). Expressive strategies in drawing are related to age and topic. Journal of Nonverbal Behavior, 31(4), 243-257. https://doi.org/10.1007/s10919-0070035-5.

Burkitt, E., Watling, D. \& Message, H. (2019). Expressivity in children's drawings of themselves for adult audiences with varied authority and familiarity. British Journal of Developmental Psychology, 37, 354-368. https://doi.org/10.1111/bjdp.12278.

Carstensen, L, L., \& DeLiema, M. (2018). The positivity effect: A negativity bias in youth fades with age. Current Opinion in Behavioral Sciences, 19, 7-12. https://doi.org/10.1016/j.cobeha.2017.07.009.

Castro, V, L., Halberstadt, A, G., \& Garrett-Peters, P. (2016). A threefactor structure of emotion understanding in third-grade children. Social Development, 25(3), 602-622. https://doi.org/10.1111/sode. 12162.

Chaplin, T, M., \& Aldao. A. (2013). Gender differences in emotion expression in children: A meta-analytic review. Psychological Bulletin, 139, 735-765. https://doi.org/10.1037/a0030737.

Cox, M, V. (1993). Children's drawings of the human figure. Erlbaum

Ekman, P., \& Friesen, W, V (2003). Unmasking the face. Malor Books

Golle, J., Mast, F, W., \& Lobmaier, J, S. (2014). Something to smile about: The interrelationship between attractiveness and emotional expression. Cognition \& Emotion, 28(2), 298-310. https://doi.org/ 10.1080/02699931.2013.817383.

Golomb, C. (2004). The child's creation of a pictorial world. Erlbaum 
Gross, A., Ballif, B. (1991). Children's understanding of emotion from facial expressions and situations: A review. Developmental Review, 11(4), 368-398. https://doi.org/10.1016/0273-2297(91)90019-K.

Gunnery, S, D., \& Ruben, M, A. (2016). Perceptions of Duchenne and non-Duchenne smiles: A meta-analysis. Cognition \& Emotion, 30(3), 501-515. https://doi.org/10.1080/02699931.2015.1018817.

Harris, D, B. (1963). Children's drawings as measures of intellectual maturity. Harcourt Brace Jovanovich

Johnson, K., Waugh, C., \& Fredrickson, B. (2010). Smile to see the forest: Facially expressed positive emotions broaden cognition. Cognition \& Emotion, 24(2), 299-321. https://doi.org/10.1080/ 02699930903384667.

LaFrance, M., Hecht, M, A., \& Levy Paluck, E, L. (2003). The contingent smile: A meta-analysis of sex differences in smiling. Psychological Bulletin, 129, 305-334. https://doi.org/10.1037/0033-2909.129.2. 305.

Laghi, F., Baiocco, R., Di Norcia, A., Cannoni, E., Baumgartner, E., \& Bombi, A, S. (2014). Emotion understanding, pictorial representations of friendship and reciprocity in school-aged children. Cognition \& Emotion, 28(7), 1338-1346. https://doi.org/10.1080/ 02699931.2014.881779.

Lau, C, Y. (2020). A cognitive learning account of the avoidance, omission and exaggerated phenomena and expressions in young adolescents' drawings of the human figure in the visual culture art education context. International Journal of Art \& Design Education, 39(3), 686-702. https://doi.org/10.1111/jade.12311.

Matto, H, C. (2007). Drawings in clinical assessment of children and adolescents. In S. R. Smith \& L. Handler (Eds) The clinical assessment of children and adolescents: A practitioner's handbook (pp. 207-221). Lawrence Erlbaum Associates Publishers.

Meng, X., Ishii, T., Sugimoto, K., Song, R., Moriguchi, Y., \& Watanabe, K. (2019). Smiling enemies: Young children better recall mean individuals who smile. Journal of Experimental Child Psychology, 188, 1-10. https://doi.org/10.1016/j.jecp.2019.104672.

Missaghi-Lakshman, M., \&Whissell, C. (1991). Children's understanding of facial expression of emotion: II. Drawing of emotion-faces. Perceptual and Motor Skills, 72, 1228-1230. https://doi.org/10. 2466/pms.1991.72.3c.1228.

Naglieri, J. A. (1988). Draw-a-person: A quantitative scoring system. Psychological Corporation

Pezzica, S., Pinto, G., Bigozzi, L., \& Vezzani, C. (2016). Where is my attention? Children's metaknowledge expressed through drawings.
Educational Psychology, 36(4), 616-637. https://doi.org/10.1080/ 01443410.2014.1003035.

Polácek, K., \& Carli, D. (1977).Test della figura umana di Goodenough e Harris: Manuale (The Goodenough-Harris test of the human figure drawing: A manual). Firenze: Organizzazioni Speciali.

Rabaglietti, E., Vacirca, M., Zucchetti, G., \& Ciairano, S. (2012). Similarity, cohesion, and friendship networks among boys and girls: A one-year follow-up study among Italian children. Current Psychology, 31(3), 246-262. https://doi.org/10.1007/s12144-0129145-2.

Sayil, M. (2001).Children's drawings of emotional faces. British Journal of Developmental Psychology, 19, 493-505. https://doi.org/10. 1348/026151001166218.

Sidera, F., Amadó, A., \& Serrat, E. (2013). Are you really happy? Children's understanding of real vs. pretend emotions. Current Psychology, 32(1), 18-31

Song, R., Over, H., \& Carpenter, M. (2016). Young children discriminate genuine from fake smiles and expect people displaying genuine smiles to be more prosocial. Evolution and Human Behavior, 37(6), 490-501. https://doi.org/10.1016/j.evolhumbehav.2016.05. 002.

Sugden, N, A., Marquis, A, R. (2017). Meta-analytic review of the development of face discrimination in infancy: Face race, face gender, infant age, and methodology moderate face discrimination. Psychological Bulletin, 143(11), 1201-1244. https://doi.org/10. 1037/bul0000116.

Talamas, S, N., Mavor, K, I., Axelsson, J., Sundelin, T., \& Perrett, D, I. (2016). Eyelid-openness and mouth curvature influence perceived intelligence beyond attractiveness. Journal of Experimental Psychology: General, 145(5), 603-620. https://doi.org/10.1037/ xge0000152.

Tuman, D, M. (2015). Gender style as form and content: An examination of gender stereotypes in the subject preference of children's drawing, Studies in Art Education, 41(1), 40-60. https://doi.org/10.1080/ 00393541.1999.11651664.

Vaish, A., Grossman, T., \& Woodward, A. (2008). Not all emotions are created equal: The negativity bias in social-emotional development. Psychological Bulletin, 134(3), 383-403. https://doi.org/10.1037/ 0033-2909.134.3.383.

Publisher's Note Springer Nature remains neutral with regard to jurisdictional claims in published maps and institutional affiliations. 